\title{
INVARIANCE OF RELATIVE INVERSE FUNCTION ORDERINGS UNDER COMPOSITIONS OF DISTRIBUTIONS
}

Abstract. Bartoszewicz and Benduch (2009) applied an idea of Lehmann and Rojo (1992) to a new setting and used the GTTT transform to define invariance properties and distances of some stochastic orders. In this paper Lehmann and Rojo's idea is applied to the class of models which is based on distributions which are compositions of distribution functions on $[0,1]$ with underlying distributions. Some stochastic orders are invariant with respect to these models.

1. Preliminaries. Let $X$ and $Y$ be two random variables, $F$ and $G$ their respective probability distribution functions and $f$ and $g$ their density functions, if they exist. Denote by $\bar{F}=1-F$ the tail (or survival function) of $F$, by $F^{-1}(u)=\inf \{x: F(x) \geq u\}, u \in(0,1)$, the quantile function and by $F^{-1}(0)$ and $F^{-1}(1)$ the lower and upper bounds of the support of $F$ respectively. We identify the distribution functions $F$ and $G$ with the respective probability distributions and denote their supports by $S_{F}, S_{G}$ respectively. We denote by $f F^{-1}$ the composition of $f$ and $F^{-1}$ and by $G^{-1} F$ the composition of $G^{-1}$ and $F$ (similarly for other densities, distributions and quantile functions). We use increasing in place of nondecreasing and decreasing in place of nonincreasing.

We deal with some stochastic orders. Let us recall their definitions and some properties for completeness. For more details we refer to Shaked and Shanthikumar (2007).

We say that $F$ is smaller than $G$ in the likelihood ratio order $\left(F \leq_{\operatorname{lr}} G\right)$ if $g(x) / f(x)$ is increasing. We say that $F$ is smaller than $G$ in the hazard rate $\operatorname{order}\left(F \leq_{\mathrm{hr}} G\right)$ if $\bar{G}(x) / \bar{F}(x)$ is increasing, or $r_{F}(x) \geq r_{G}(x)$ for every $x$ if

2010 Mathematics Subject Classification: 60E05, 60E15, $62 \mathrm{~N} 05$.

Key words and phrases: convex order, dispersive order, star order, superadditive order, orbits, general order statistics, maximal invariant, distance between distribution functions. 
$F$ and $G$ are absolutely continuous, where $r_{F}(x)=f(x) / \bar{F}(x)$ is the hazard rate function of $F$. We say that $F$ is smaller than $G$ in the reversed hazard rate order $\left(F \leq_{\mathrm{rh}} G\right)$ if $G(x) / F(x)$ is increasing, or $\breve{r}_{F}(x) \leq \breve{r}_{G}(x)$ for every $x$ if $F$ and $G$ are absolutely continuous, where $\breve{r}_{F}(x)=f(x) / F(x)$ is the reversed hazard rate function of $F$. We say that $F$ is stochastically smaller than $G\left(F \leq_{\text {st }} G\right)$ if $F(x) \geq G(x)$ for every $x$, or equivalently $\bar{F}(x) \leq \bar{G}(x)$ for every $x$.

Lehmann and Rojo (1992) noticed that these stochastic orders may be defined by properties of the function $k(u)=G F^{-1}(u), u \in(0,1): F \leq_{\operatorname{lr}} G$ $\Leftrightarrow k(u)$ is convex; $F \leq_{\mathrm{hr}} G \Leftrightarrow 1-k^{-1}(1-u)$ is star-shaped; $F \leq_{\mathrm{rh}} G \Leftrightarrow$ $k(u)$ is star-shaped; $F \leq_{\text {st }} G \Leftrightarrow k(u) \leq u$ for all $u \in(0,1)$.

It is also well known that

$$
\begin{array}{ccc}
F \leq_{\mathrm{lr}} G \Rightarrow & F \leq_{\mathrm{hr}} G \\
\Downarrow & \Downarrow \\
F \leq_{\mathrm{rh}} G \Rightarrow & F \leq_{\mathrm{st}} G
\end{array}
$$

We shall also consider so called relative inverse function orderings; see Müller and Stoyan (2002) and van Zwet (1964). They are defined by properties of the function $G^{-1} F$. For continuous distributions the graph of the relative inverse distribution function is identical with the so-called Q-Q plot.

We say that $F$ is smaller than $G$ in the dispersive $\operatorname{order}\left(F \leq_{\operatorname{disp}} G\right)$ if $G^{-1} F$ is dispersive, i.e. $G^{-1} F(x)-x$ is increasing.

Let now $X$ and $Y$ be positive random variables. We say that $F$ is smaller than $G$ in the convex transform order $\left(F \leq_{\mathrm{c}} G\right)$ if $G^{-1} F$ is convex on $S_{F}$. We say that $F$ is smaller than $G$ in the star order $\left(F \leq_{*} G\right)$ if $G^{-1} F$ is starshaped on $S_{F}$, i.e. $G^{-1} F(x) / x$ is increasing in $x>0$. We say that $F$ is smaller than $G$ in the superadditive order $\left(F \leq_{\text {su }} G\right)$ if $G^{-1} F$ is superadditive, i.e. $G^{-1} F(x+y) \geq G^{-1} F(x)+G^{-1} F(y), x \geq 0$ for $y \geq 0$.

It is well known (see Shaked and Shanthikumar, 2007) that

$$
F \leq_{\mathrm{c}} G \Rightarrow F \leq_{*} G \Rightarrow F \leq_{\mathrm{su}} G .
$$

If $-\infty<F^{-1}(0)=G^{-1}(0)$, then

$$
F \leq_{\text {disp }} G \Rightarrow F \leq_{\text {st }} G .
$$

Lehmann and Rojo (1992) characterized stochastic orders defined by properties of $G F^{-1}$ in terms of invariance under monotone transformations. Bartoszewicz and Benduch (2009) gave a characterization of relative inverse function orders in terms of invariance under the GTTT transform with respect to a class of positive continuous functions on $(0,1)$. In this note, Lehmann and Rojo's idea is applied to a new setting. Compositions of some distributions on $[0,1]$ with underlying distributions are used to define invariance properties and distances of some stochastic orders. 


\section{Results}

2.1. Invariant preorderings. In this section we consider the convex, star-shaped, dispersive, superadditive and common stochastic orders.

Let $\mathcal{F}$ denote the class of continuous distributions on $[0, \infty)$ and let $\boldsymbol{\Psi}$ be the class of continuous strictly increasing distribution functions on $[0,1]$. It is easy to see that $\boldsymbol{\Psi}$ is a group with respect to composition. Also, for every $F \in \mathcal{F}$ and every $\psi \in \mathbf{\Psi}, \psi F \in \mathcal{F}$.

The distribution functions $\psi F$ with $\psi$ increasing, not necessarily strictly increasing, $\psi(0)=0$ and $\psi(1)=1$ are called distorted distributions and have been studied in different areas. Wang and Young (1998) connected these types of distributions with stochastic orders. Recently, Sordo and SuarezLlorens (2008) and Khaledi and Shaked (2010) obtained some new results about stochastic orderings of distorted distributions. Navarro and Rychlik (2010) obtained some bounds and showed that the distributions of coherent systems with independent and identically distributed components are also distorted distributions obtained from the common component distribution. There are many examples of stochastic models where the probability distributions under consideration are of the form $\psi F \in \mathcal{F}, \psi \in \boldsymbol{\Psi}$. Here we list some of them.

EXAMPLE 1 (Generalized order statistics). Kamps (1995) introduced uniform generalized order statistics. Let $n \in \mathbb{N}, k \geq 1, m_{1}, \ldots, m_{n-1} \in \mathbb{R}$, $M_{r}=m_{r}+\cdots+m_{n-1}$, be parameters such that $\gamma_{r}=k+n-r+M_{r} \geq 1$ for all $r \in\{1, \ldots, n-1\}$ and let $\tilde{m}=\left(m_{1}, \ldots, m_{n-1}\right)$ if $n \geq 2(m \in \mathbb{R}$ arbitrary if $n=1)$. If random variables $U(r, n, \tilde{m}, k), r=1, \ldots, n$, possess a joint density function of the form

$$
\begin{aligned}
f^{U(1, n, \tilde{m}, k), \ldots, U(n, n, \tilde{m}, k)}\left(u_{1}, \ldots, u_{n}\right) & \\
& =k\left[\prod_{j=1}^{n-1} \gamma_{j}\right]\left[\prod_{i=1}^{n-1}\left(1-u_{i}\right)^{m_{i}}\right]\left(1-u_{n}\right)^{k-1}
\end{aligned}
$$

on the cone $\left\{\left(u_{1}, \ldots, u_{n}\right): 0 \leq u_{1} \leq \cdots \leq u_{n} \leq 1\right\} \subset \mathbb{R}^{n}$, then they are called uniform generalized order statistics.

Let $F$ be a distribution function. The random variables

$$
X(r, n, \tilde{m}, k)=F^{-1}(U(r, n, \tilde{m}, k)), \quad r=1, \ldots, n,
$$

are called generalized order statistics based on the distribution function $F$.

It is clear that the distribution function of $\tilde{X}=X(r, n, \tilde{m}, k)$ is of the form

$$
F_{\tilde{X}}(x)=\psi_{r, n, \tilde{m}, k} F(x),
$$

where $\psi_{r, n, \tilde{m}, k} \in \boldsymbol{\Psi}$ is the distribution function of $U(r, n, \tilde{m}, k)$. 
Choosing appropriate parameters in (1) we obtain among others the following models: ordinary order statistics of a sample of size $n$, order statistics with non-integral sample size, sequential order statistics, record values, $k$ th record values, Pfeifer's record values (see Kamps, 1995). We present here two particular important cases.

1. Order statistics. Let $X_{1: n}, X_{2: n}, \ldots, X_{n: n}$ be order statistics of a sample of size $n$ from the distribution $F$. It is well known that the distribution function of $X_{i: n}, i=1, \ldots, n$, is of the form

$$
F_{i: n}(x)=B_{i: n} F(x),
$$

where $B_{i: n}$ is the distribution function of the beta distribution $B(i, n-i+1)$. Obviously, $B_{i: n} \in \boldsymbol{\Psi}$.

Extensions of order statistics to those with non-integral sample size are due to Stiegler (1977), Rohatgi and Saleh (1988) provide another example of models with distributions from $\mathbf{\Psi}$.

2. Record values. Let $\left\{X_{i}\right\}_{i \in \mathbb{N}}$ be a sequence of iid random variables with an absolutely continuous distribution $F$ and density $f$. The random variables $L(n), n \in \mathbb{N}$, defined by $L(1)=1$ and $L(n+1)=\min \{j>L(n)$; $\left.X_{j}>X_{L(n)}\right\}$, are called record times and $X_{L(n)}, n \in \mathbb{N}$, are called upper record values. The marginal density of $L(r)$ is of the form

$$
f_{X_{L(r)}}(x)=\frac{1}{(r-1) !}\left[\log \frac{1}{1-F(x)}\right]^{r-1} f(x)
$$

and the marginal distribution function is given by

$$
F_{X_{L(r)}}(x)=1-[1-F(x)] \sum_{j=0}^{r-1} \frac{1}{j !}\left[\log \frac{1}{1-F(x)}\right]^{j},
$$

i.e.

$$
F_{X_{L(r)}}(x)=\psi_{r} F(x),
$$

where

$$
\psi_{r}(t)=1-(1-t) \sum_{j=0}^{r-1} \frac{1}{j !}\left[\log \frac{1}{1-t}\right]^{j}, \quad t \in[0,1] .
$$

Similarly, the distribution of the $k$ th record value (see Dziubdziela and Kopociński (1976) or Kamps (1995) for the definition) is of the form

$$
F_{X_{L(r)_{k}}}(x)=\psi_{r, k} F(x),
$$

where

$$
\psi_{r, k}(t)=1-(1-t)^{k} \sum_{j=0}^{r-1} \frac{1}{j !}\left[k \log \frac{1}{1-t}\right]^{j}, \quad t \in[0,1] .
$$

Obviously, $\psi_{r}$ and $\psi_{r, k}$ are distribution functions from $\boldsymbol{\Psi}$. 
EXAMPLE 2 (Models in survival analysis). Three models are of great importance in survival analysis (we give the corresponding probability distribution functions):

1. Proportional hazard model

$$
\psi_{\alpha} F(x)=1-[1-F(x)]^{\alpha}, \quad x \in \mathbb{R}, \alpha>0,
$$

i.e.

$$
\psi_{\alpha}(t)=1-(1-t)^{\alpha}, \quad t \in[0,1] .
$$

2. Proportional reversed hazard model

$$
\psi_{\alpha} F(x)=[F(x)]^{\alpha}, \quad x \in \mathbb{R}, \alpha>0,
$$

i.e.

$$
\psi_{\alpha}(t)=t^{\alpha}, \quad t \in[0,1] .
$$

3. Proportional odds model

$$
\psi_{\alpha} F(x)=1-\frac{\alpha(1-F(x))}{1-(1-\alpha)(1-F(x))}, \quad x \in \mathbb{R}, \alpha>0,
$$

i.e.

$$
\psi_{\alpha}(t)=1-\frac{\alpha(1-t)}{1-(1-\alpha)(1-t)}, \quad t \in[0,1] .
$$

One can also consider the two-parameter proportional odds model with

$$
\psi_{\alpha, c}(t)=1-\left[\frac{\alpha(1-t)^{c}}{1-(1-\alpha)(1-t)^{c}}\right]^{1 / c}, \quad t \in[0,1], \alpha>0, c>0 .
$$

Of some importance are exponential mixtures of distributions in the proportional hazard model and the proportional reversed hazard model (see Marshall and Olkin, 1997). If the parameter $\alpha$ has the exponential distribution $K(\alpha)=1-e^{-\theta \alpha}, \alpha>0, \theta>0$, then we obtain the following distributions from $\boldsymbol{\Psi}$ :

$$
\begin{aligned}
& \psi_{\theta}(t)=\frac{\theta}{\theta-\log t}, \quad t \in(0,1), \\
& \psi_{\theta}(t)=1-\frac{\theta}{\theta-\log (1-t)}, \quad t \in(0,1),
\end{aligned}
$$

for the proportional hazard model and the proportional reversed hazard model respectively.

The proportional hazard model is well known, it was introduced and studied by Cox (1972). The proportional reversed hazard model was extensively studied by Di Crescenzo (2000) Kirmani and Gupta (2001) considered the proportional odds model; see also Marshall and Olkin (1997) and BenduchFrąszczak (2010), The two-parameter proportional odds model was studied by Dąbrowska and Doksum (1988). 
Define a preorder in $\mathcal{F}$ to be any set $\mathcal{S}$ of ordered pairs $(F, G)$ in $\mathcal{F} \times \mathcal{F}$ satisfying

$$
\begin{gathered}
(F, F) \in \mathcal{S} \quad \text { for all } F \in \mathcal{F}, \\
(F, G) \in \mathcal{S} \text { and }(G, H) \in \mathcal{S} \quad \text { implies } \quad(F, H) \in \mathcal{S} .
\end{gathered}
$$

When $(F, G) \in \mathcal{S}$, we write $F \leq_{S} G$.

Definition 1. The preorder $\mathcal{S}$ is invariant under the class $\boldsymbol{\Psi}$ if

$$
F \leq_{S} G \Rightarrow \psi F \leq_{S} \psi G \text { for all } \psi \in \boldsymbol{\Psi} .
$$

Similarly to Lehmann and Rojo (1992) we define orbits of pairs $(F, G)$ under transformations $\psi$ from $\mathbf{\Psi}$ in the space $\mathcal{F} \times \mathcal{F}$,

$$
\mathcal{O}(F, G)=\{(\psi F, \psi G): \psi \in \mathbf{\Psi}\} .
$$

Thus $(F, G) \in \mathcal{S}$ if and only if the orbit $\mathcal{O}(F, G)$ is contained in $\mathcal{S}$. Also as in Lehmann and Rojo (1992) we label the orbits by a maximal invariant under the group $\boldsymbol{\Psi}$. We prove the following:

THEOREM 1. The function

$$
\lambda(u)=G^{-1} F(t), \quad t>0,
$$

is a maximal invariant under the group $\mathbf{\Psi}$.

Proof. It is easy to notice that $(\psi G)^{-1} \psi F=G^{-1} F$ for every $\psi \in \mathbf{\Psi}$, i.e. $G^{-1} F$ is invariant under the group $\boldsymbol{\Psi}$.

Let $\left(F_{1}, G_{1}\right)$ and $\left(F_{2}, G_{2}\right)$ be two pairs of distributions from $\mathcal{F}$ and let

$$
G_{1}^{-1} F_{1}(t)=G_{2}^{-1} F_{2}(t), \quad t>0 .
$$

We should prove that $\left(F_{1}, G_{1}\right)$ and $\left(F_{2}, G_{2}\right)$ are in the same orbit, i.e. there exists a function $\psi_{0} \in \boldsymbol{\Psi}$ such that $F_{2}(t)=\psi_{0} F_{1}(t)$, for $G_{1}$ and $G_{2}$ analogously. Since (5) holds, we have also

$$
G_{2} G_{1}^{-1}(t)=F_{2} F_{1}^{-1}(t), \quad t>0,
$$

and then we put

$$
\psi_{0}(t)=G_{2} G_{1}^{-1}(t)=F_{2} F_{1}^{-1}(t), \quad t>0 .
$$

Thus we have $\psi_{0} F_{1}(t)=F_{2} F_{1}^{-1} F_{1}(t)=F_{2}(t)$ and $\psi_{0} G_{1}(t)=G_{2} G_{1}^{-1} G_{1}(t)$ $=G_{2}(t)$.

From Theorem 1 and the definitions of the relevant stochastic orders we obtain

COROllary 1. The convex, star, superadditive, dispersive and common stochastic orders are invariant under the group $\mathbf{\Psi}$.

Denote by $\boldsymbol{\Lambda}_{S}$ the class of functions of the form (4) which are maximal invariants for the preorder $S$ under the group $\boldsymbol{\Psi}$. From Corollary 1 it follows that $\boldsymbol{\Lambda}_{S}$ is the class of positive increasing convex functions if $S$ is the convex 
transform order; the class of positive increasing star-shaped functions if $S$ is the star order; the class of dispersive functions if $S$ is the dispersive order, etc. It follows from transitivity and symmetry of the order that $\boldsymbol{\Lambda}_{S}$ is closed under composition and contains the identity function.

2.2. Ordering the orbits and distances between ordered distributions. The following idea of Lehmann and Rojo (1992) (see also Bartoszewicz and Benduch, 2009) may also be applied to some relative inverse function orderings. They considered quadruples $\left(F_{1}, G_{1} ; F_{2}, G_{2}\right)$ and used invariant preorderings to define the concept of $G_{2}$ being further to the right from $F_{2}$ than $G_{1}$ is of $F_{1}$. Let us repeat their considerations in our setting.

Consider a quadruple $\left(F_{1}, G_{1} ; F_{2}, G_{2}\right)$ such that $F_{1}, G_{1} \in \mathcal{F}, F_{2}=\psi F_{1}$ and $G_{2}=\psi G_{1}$ for some $\psi \in \boldsymbol{\Psi}$. Since $\left(F_{1}, G_{1}\right)$ and $\left(F_{2}, G_{2}\right)$ are in the same orbit, we say that $F_{1}$ and $G_{1}$ are at the same distance from each other as $F_{2}$ and $G_{2}$, i.e. $d\left(F_{1}, G_{1}\right)=d\left(F_{2}, G_{2}\right)$, where $d$ is a function which measures the distance if such a function exists. On the other hand, if $F_{1} \leq_{S} G_{1} \leq_{S} G_{2}$, we say that $G_{2}$ is further to the right from $F_{1}$ than $G_{1}$ is.

One now asks when for any quadruple $\left(F_{1}, G_{1} ; F_{2}, G_{2}\right)$ the distribution $G_{2}$ will be further to the right from $F_{2}$ than $G_{1}$ is of $F_{1}$ in the preorder $S$. Let $F_{1}, G_{1} \in \mathcal{F}$ and $F_{i} \leq_{S} G_{i}, i=1,2$. Notice that the function $\psi_{0}=F_{2} F_{1}^{-1} \in \Psi$ has the property that $F_{2}=\psi_{0} F_{1}$. Therefore $\psi_{0} G_{1}=F_{2} F_{1}^{-1} G_{1}$ and $G_{2}$ is further to the right from $F_{2}$ than $G_{1}$ is of $F_{1}$ in the preorder $S$ if

$$
\psi_{0}\left(G_{1}\right) \leq_{S} G_{2}
$$

i.e.

$$
G_{2}^{-1} \psi_{0} G_{1}=G_{2}^{-1} F_{2} F_{1}^{-1} G_{1}=G_{2}^{-1} F_{2}\left(G_{1}^{-1} F_{1}\right)^{-1} \in \boldsymbol{\Lambda}_{S}
$$

Denote $\lambda_{i}=G_{i}^{-1} F_{i}, i=1,2$. We may formulate the following definition.

Definition 2. Let $F_{1} \leq_{S} G_{1}, F_{2} \leq_{S} G_{2}$. Then $G_{2}$ is said to be further to the right from $F_{2}$ than $G_{1}$ is of $F_{1}$ if

$$
\lambda_{2} \lambda_{1}^{-1} \in \boldsymbol{\Lambda}_{S}
$$

The following examples illustrate this concept.

ExAmple 3. Consider the scale family $F(t / \theta), \theta>0$, where $F \in \mathcal{F}$ is fixed, and define $G_{1}(t)=F\left(t / \theta_{1}\right)$ and $G_{2}(t)=F\left(t / \theta_{2}\right)$ with $\theta<\theta_{1}<\theta_{2}$. Obviously, $F \leq_{\operatorname{disp}} G_{1}$ and $F \leq_{\text {disp }} G_{2}$. Let $\lambda_{i}=G_{i}^{-1} F, i=1,2$. It is easy to see that

$$
\lambda_{2} \lambda_{1}^{-1}(t)=G_{2}^{-1} F F^{-1} G_{1}(t)=\frac{\theta_{2}}{\theta_{1}} t
$$

is dispersive. According to Definition 2, $G_{2}$ is further to the right from $F$ than $G_{1}$ is in dispersive ordering. 
EXAMPLE 4 . Let $F_{\alpha}$ be the beta distribution with density

$$
f_{\alpha}(t)=\alpha(1-t)^{\alpha-1}, \quad \alpha>0,0<t<1 .
$$

Then $F_{\alpha}^{-1}(t)=1-(1-t)^{1 / \alpha}$. Notice that $F_{\beta}^{-1} F_{\alpha}(t)=1-(1-t)^{\alpha / \beta}$ is convex if $\alpha<\beta$. Therefore $F_{\alpha} \leq_{\mathrm{c}} F_{\beta}$ and $F_{\delta} \leq_{\mathrm{c}} F_{\gamma}$ if $0 \leq \alpha<\beta$ and $0 \leq \delta<\gamma$. Then $F_{\gamma}^{-1} F_{\delta}\left(F_{\beta}^{-1} F_{\alpha}\right)^{-1}(t)=1-(1-t)^{\beta \delta / \alpha \gamma}$ is convex if $\alpha \gamma / \beta \delta \geq 1$. Hence if $\alpha / \beta \geq \delta / \gamma$, then $F_{\gamma}$ is further to the right from $F_{\delta}$ than $F_{\beta}$ is of $F_{\alpha}$ in the convex ordering.

EXAmPle 5. Consider the distribution functions

$$
F_{i}(t)=1-e^{-t^{\beta_{i}}}, \quad \beta_{i}>0, i=1,2, \quad \text { and } \quad G(t)=1-e^{-t}, \quad t \geq 0 .
$$

with the quantile functions of the respective forms

$$
F_{i}^{-1}(t)=-\log (1-t)^{1 / \beta_{i}}, \quad G^{-1}(t)=-\log (1-t) .
$$

It is well known that for $\beta_{i} \geq 1, i=1,2$, the distributions $F_{1}$ and $F_{2}$ are IFR and $F_{i} \leq_{\mathrm{c}} G, i=1,2$. We compute

$$
F_{1}^{-1} F_{2}(t)=t^{\beta_{2} / \beta_{1}} \text {. }
$$

The function (7) is convex if $\beta_{2} / \beta_{1} \geq 1$. Thus if $\beta_{2} \geq \beta_{1}$, then $G$ is further to the right from $F_{2}$ than $F_{1}$ is in the convex transform ordering as well as in the star ordering.

One may ask whether there exists a metric $d(F, G)$ which is consistent with the above comparison of distributions. Since $\lambda$-functions are invariant under the function $\psi \in \mathbf{\Psi}$, the distance $d(F, G)$ must also be invariant under these transforms. Therefore such a metric must satisfy the following two conditions:

(A) $d(F, G)=d(\psi F, \psi G)$ for all $\psi \in \mathbf{\Psi}$;

(B) $d\left(F_{1}, G_{1}\right) \leq d\left(F_{2}, G_{2}\right)$ if (7) holds.

THEOREM 2. Let $F$ and $G$ be distributions from $\mathcal{F}$ such that $F^{-1}(0)=$ $G^{-1}(0)=0$. If $S$ is the dispersive order, then the distance

$$
d_{*}(F, G)=\sup _{t \in(0,1)}\left|G^{-1}(t)-F^{-1}(t)\right|=\sup _{x}\left|G^{-1} F(x)-x\right|
$$

satisfies conditions $(\mathrm{A})$ and $(\mathrm{B})$.

Proof. It is evident that $d_{*}$ satisfies (A).

The condition (8) is equivalent to the fact that $G_{2}^{-1} F_{2} F_{1}^{-1} G_{1}$ is dispersive, that is, $G_{2}^{-1} F_{2} F_{1}^{-1} G_{1}(x)-x$ is increasing. From the assumption we have $G_{2}^{-1} F_{2} F_{1}^{-1} G_{1}(x)-x \geq 0$ and then $G_{2}^{-1} F_{2}(x)-x \geq G_{1}^{-1} F_{1}(x)-x \geq 0$, $x \geq 0$. Hence

$$
d_{*}\left(F_{1}, G_{1}\right) \leq d_{*}\left(F_{2}, G_{2}\right)
$$


ExAmple 6 . Let $\bar{F}(x)=\frac{1}{2}\left(e^{-\alpha x}+e^{-x}\right), \alpha>1$, and $\bar{G}(x)=e^{-x}$. Then

$$
\begin{aligned}
G^{-1} F(x)-x & =\log \left(\frac{2}{e^{-\alpha x}+e^{-x}}\right)-x=\log \left(\frac{2 e^{x}}{e^{-(\alpha+1) x}+1}\right)-\log e^{x} \\
& =\log \left(\frac{2}{e^{-(\alpha+1) x}+1}\right)
\end{aligned}
$$

is increasing and hence $F \leq_{\text {disp }} G$. Therefore we obtain the distance $d_{*}$ between the distributions $F$ and $G$,

$$
d_{*}(F, G)=\sup _{x}\left|G^{-1} F(x)-x\right|=\sup _{x}\left|\log \left(\frac{2}{e^{-(\alpha+1) x}+1}\right)\right|=\log 2 .
$$

Acknowledgments. Research of M. Frąszczak is supported by Ministry of Science and Higher Education, Poland, Grant N N201 363836.

\section{References}

\J. Bartoszewicz and M. Benduch (2009), Some properties of the generalized TTT transform, J. Statist. Plann. Inference 139, 2208-2217.

M. Benduch-Frąszczak (2010), Some properties of the proportional odds model, Appl. Math. (Warsaw) 37, 247-256.

D. R. Cox (1972), Regression models and life tables, J. R. Statist. Soc. Ser. B 34, 187-202.

$\square$ D. M. Dąbrowska and K. Doksum (1988), Estimation and testing in a two-sample generalized odds-rate model, J. Amer. Statist. Assoc. 83, 744-749.

\rceil A. Di Crescenzo (2000), Some results on the proportional reversed hazards model, Statist. Probab. Lett. 50, 313-321.

W. Dziubdziela and B. Kopociński (1976), Limiting properties of the k-record values, Zastos. Mat. 15, 187-190.

U. Kamps (1995), A Concept of Generalized Order Statistics, Teubner, Stuttgart.

B. E. Khaledi and M. Shaked (2010), Stochastic comparisons of multivariate mixtures, J. Multivariate Anal. 101, 2486-2498.

S. N. U. A. Kirmani and R. C. Gupta (2001), On the proportional odds model in survival analysis, Ann. Inst. Statist. Math. 53, 203-216.

E. L. Lehmann and J. Rojo (1992), Invariant directional orderings, Ann. Statist. 20, 2100-2110.

]A. W. Marshall and I. Olkin (1997), A new method for adding a parameter to a family of distributions with application to the exponential and Weibull families, Biometrika 84, 641-652.

A. Müller and D. Stoyan (2002), Comparison Methods for Stochastic Models and Risks, Wiley, New York.

J. Navarro and T. Rychlik (2010), Comparisons and bounds for expected lifetimes of reliability systems, Eur. J. Oper. Res. 207, 309-317.

V. K. Rohatgi and A. K. M. D. E. Saleh (1988), A class of distributions connected to order statistics with nonintegral sample size, Comm. Statist. Theory Methods 17, 2005-2012.

M. Shaked and J. G. Shanthikumar (2007), Stochastic Orders, Springer, New York.

$\square$ M. A. Sordo and A. Suarez-Llorens (2011), Stochastic comparisons of distorted variability measures, Insurance Math. Econom. 49, 11-17. 
S. M. Stiegler (1977), Fractional order statistics, with applications, J. Amer. Statist. Assoc. 72, 544-550.

W. R. van Zwet (1964), Convex Transformations of Random Variables, Mathematisch Centrum, Amsterdam.

S. Wang and V. R. Young (1998), Ordering risks: Expected utility theory versus Yaari's dual theory of risk, Insurance Math. Econom. 22, 145-161.

Magdalena Frąszczak

Institute of Genetics

Wrocław University of Environmental and Life Sciences

7 Kożuchowska St.

51-631 Wrocław, Poland

E-mail: magdalena.fraszczak@up.wroc.pl
Jarosław Bartoszewicz Mathematical Institute University of Wrocław Pl. Grunwaldzki 2/4 50-384 Wrocław, Poland E-mail: jarbar@math.uni.wroc.pl

Received on 24.5.2012;

revised version on 3.8.2012 